Voix et Images

\title{
D’abord l'intime. Entretien avec Denise Desautels
}

\section{Louise Dupré}

Volume 26, numéro 2 (77), hiver 2001

Denise Desautels

URI : https://id.erudit.org/iderudit/201536ar

DOI : https://doi.org/10.7202/201536ar

Aller au sommaire du numéro

Éditeur(s)

Université du Québec à Montréal

ISSN

0318-9201 (imprimé)

1705-933X (numérique)

Découvrir la revue

Citer ce document

Dupré, L. (2001). D’abord l'intime. Entretien avec Denise Desautels. Voix et

Images, 26(2), 227-240. https://doi.org/10.7202/201536ar d'utilisation que vous pouvez consulter en ligne.

https://apropos.erudit.org/fr/usagers/politique-dutilisation/ 


\title{
D'abord l'intime. Entretien avec Denise Desautels
}

\author{
Louise Dupré, Université du Québec à Montréal
}

V. et I.: Votre récit, Ce fauve, le Bonbeur, porte sur votre enfance. Or, comme beaucoup de familles modestes de l'époque, on sent que votre famille n'a pas cherché à développer chez vous le goût de l'écriture. D'où vous est venu votre désir d'écrire?

D. D.: Ce désir est apparu à l'adolescence, au moment où les multiples morts de mon enfance, longtemps refoulées, tenues à l'écart de la conscience - à cause de cette nécessité absolue du Bonheur, qui s'était imposée dans ma famille - , ont fini par se frayer un chemin à l'intérieur de moi. Mais, comme tout se passait dans l'ombre, j'ai été une adolescente souvent triste et mélancolique, aux prises avec une émotion exacerbée que je ne comprenais pas. En réalité, j'étais sournoisement rattrapée par une multitude de deuils qu'on ne m'avait pas appris à faire, qu'on avait simplement camouflés sous un lot de bercements et de caresses, en se servant de la vie éternelle comme consolation. À quatorze ou quinze ans, j'ai commencé à écrire des poèmes et des petits textes en prose dans des cahiers noirs à couverture rigide, que je traînais partout avec moi. J'avais besoin de mettre en mots cet excès d'émotion qui me paralysait. Il y avait beaucoup de naïveté dans ces textes: je m'y épanchais, y répandais ma tristesse avec complaisance, sans chercher à la percer. À la même époque, je rêvais d'être pianiste, peintre, danseuse, comédienne. Tous les arts et les artistes m'attiraient. Peut-être avais-je l'intuition que c'est de là que me viendrait l'éclaircie. Or, je n'aurai aucune précocité, je mettrai des années à saisir le sens de cette première intuition, à saisir aussi que c'est du côté de la littérature que me viendrait cette éclaircie.

V. et I. : Vous avez une formation en littérature, je crois...

D. D. : J'ai fait une licence en lettres et une maîtrise en création littéraire à l'Université de Montréal. La promeneuse et l'oiseau suivi du Journal de la promeneuse, le texte de mon mémoire que Pierre Nepveu a dirigé, a été publié aux Éditions du Noroît en 1980. 
V. et I.: Votre récit se termine sur une scène où la protagoniste se retrouve face à une exposition de Van Gogh. Votre intérêt pour les arts visuels semble être venu très tôt...

D. D.: J'aurais aimé savoir dessiner, peindre, sculpter. Ma mère avait ce talent, qu'elle n'a jamais développé ni pris au sérieux, de transformer les matériaux qui lui tombaient sous la main - cire, papier métallique, papier de soie, fil de plomb, etc. - en petites sculptures. Elle a aussi fait quelques croquis au crayon. J'en ai conservé un, tout petit, qui m'émeut encore, où l'on me voit, à quinze ans peut-être, les cheveux longs, bien installée sur un tabouret, le dos au mur, les jambes relevées, appuyées sur le chambranle de la porte, en train de parler au téléphone. L'habileté de ma mère me fascinait et, en même temps, me ramenait à ma propre incompétence. Au collège Basile-Moreau, je me suis inscrite à des cours de dessin et j'ai suivi par plaisir deux cours d'histoire de l'art, Moyen Âge èt Renaissance, qui m'ont donné le goût de continuer dans cette voie. À l'université, j'ai fait, avec François-Marc Gagnon et Bernard Tesseydre, entre autres, un certificat en histoire de l'art. J'ai parfois eu l'impression d'être allée vers l'écriture parce que ça allait de soi, que j'étais bonne en français et en composition, comme on le disait à l'époque, alors que je n'avais aucun talent, ni pour la peinture ni pour la musique. Aujourd'hui je sais que cette descente au coeur de la langue, cette continuelle quête de sens, qui fait bouger à la fois l'émotion, la pensée et l'écriture, en nous ramenant forcément vers nous-mêmes, m'est esséntielle et que, par conséquent, ce choix n'était peut-être pas aussi arbitraire que je le croyais à l'époque.

V. et I. : Par la suite, vous avez collaboré avec des artistes. Qu'est-ce que ces collaborations ont apporté à votre travail?

D. D.: J'aime penser que les œuvres des artistes avec lesquels j'ai collaboré m'ont permis de déplacer mon imaginaire, qu'elles l'ont secoué, m'entraînant le plus souvent sur des chemins de traverse que, seule, laissée à moi-même, je n'aurais peut-être pas eu l'audace de prendre. Quand on se présente comme une poète préoccupée par la mémoire et les blessures qui s'y sont accumulées, une sorte d'archéologue de l'intime, et qu'on écrit beaucoup, depuis plusieurs années déjà, on risque fort, malgré un ardent désir de lucidité, de se piéger soi-même, de contourner avec habileté, et malgré une bonne foi indéniable, ce qui pourrait être trop périlleux, de poursuivre son chemin comme si de rien n'était, et au bout du compte de se répéter, de ronronner, avec l'illusion de l'approfondissement. Comme poète, il me semble qu'on est toujours en danger de répétition. De piétinement. Avec le temps, on arrive à se créer un langage, un univers, un style, quelque chose de précieux avec lequel on se sent à l'aise et qu'on devrait pouvoir pousser toujours plus loin, transformer à sa guise. Or, je n'ai jamais été sûre d'avoir cette aptitude naturelle pour la métamorphose. 
Mon désir m'emporte de ce côté-là, mais ma fragilité et mes doutes sont parfois plus forts que mon désir. En choisissant d'aller vers des ouvres d'art, souvent exigeantes, porteuses des grandes inquiétudes humaines, je choisis d'une certaine manière de ne pas fuir, de contourner cette fois non pas le péril lui-même mais la peur du péril, et d'ouvrir ainsi ma petite mémoire intime à d'autres mémoires, avec toutes leurs strates de souvenirs désordonnés, enchevêtrés, douloureux, souvent obscurs, souvent même tout à fait opaques, enfermés sous un faux apaisement de surface. Je dois avouer que mes intrusions en terrain miné sont beaucoup moins téméraires qu'elles ne paraissent l'être puisque les artistes m'y ont précédée, y ont laissé leurs marques. Il serait plus juste de dire que je m'aventure sur un terrain déjà déminé, inscrit dans un projet, métamorphosé par l'art, et que j'y avance avec la sensation d'être accompagnée.

V. et I. : Je vois chez vous plusieurs types de collaboration: les recueils illustrés par un artiste, les recueils faits à partir d'œuvres visuelles, les livres d'artiste, parfois les catalogues d'exposition. Y a-t-il des postures d'écriture différentes qui répondent à chacune de ces collaborations?

D. D. : En effet, les postures d'écriture varient selon les types de collaboration. Les quatre ou cinq premiers recueils, que j'ai publiés aux Éditions du Noroît, n'ont à peu près rien à voir avec ce qu'on pourrait appeler des œuvres en collaboration. À cette époque, comme cela se passait habituellement au Noroît, je présentais des manuscrits déjà achevés à des artistes dont j'aimais le travail et auxquels je demandais deux ou trois images d'accompagnement parce que leur univers me semblait, d'une certaine manière, recouper le mien. Mais, à partir de 1986, avec la parution de $L a$ répétition et de Écritures/Ratures, mon attitude s'est transformée. Dans le premier cas, je me suis souvenue, en cours de rédaction, de l'installation d'Irene $\mathrm{F}$. Whittome, La salle de classe, présentée au Musée des beaux-arts de Montréal, en 1980. Je suis donc entrée en contact avec l'artiste bien avant d'avoir terminé le manuscrit, et notre rencontre, et les photographies de l'œuvre, qu'elle a mises à ma disposition, ont marqué la suite des événements. J'ai écrit La répétition en mêlant librement mon univers au sien. Déjà, à ce moment-là, j'avais été approchée par Francine Simonin, qui souhaitait que nous retravaillions ensemble à un projet de vraie collaboration, dans lequel s'inséreraient des dessins préparatoires aux tableaux et des esquisses de textes. Après ces deux expériences, par ailleurs éloignées l'une de l'autre, le rapport entre mon écriture et les arts visuels n'a plus jamais été le même. Et, pour la première fois en 1988, c'est moi qui ai pris les devants, allant à la rencontre de Michel Goulet et des sculptures qu'il présentait à la Biennale de Venise. Sans doute y avait-il beaucoup d'inconscience dans l'enthousiasme qui me portait vers cet univers masculin autour duquel j'allais articuler le mien. Pourtant je ne l'ai jamais regretté. Leçons de Venise m'a donné la chance de mettre en écriture - ce qui n'avait peut-être été jusque-là qu'une intuition - les liens troublants 
et pourtant subtils qui existent entre l'intime et le politique. Tout compte fait, je crois que ce livre a secoué ma langue et mon imaginaire, leur insufflant un étrange dynamisme. D'autres expériences similaires, à partir d'œuvres souvent fondamentalement différentes les unes des autres celles de Martha Townsend, Peter Krausz, Betty Goodwin, Monique Bertrand, Alain Laframboise, entre autres -, m'ont permis de m'éloigner de mes obsessions d'écrivaine, puis d'y revenir avec un regard autre, à la fois plus ample et plus juste. Du moins, je l'espère. En ce qui concerne les livres d'artiste, dans la mesure où on y retrouve souvent de courts textes intimement liés à un objet-livre conçu, en grande partie, hors de la salle de travail, tout y repose sur une vraie complicité entre un concepteur, un artiste et un écrivain. C'est avec Jacques Fournier, directeur des Éditions Roselin, que j'ai appris le vrai sens de ce type de travail en collaboration, plus près de ce qui se passe au théâtre ou en musique qu'en littérature. Ces expériences viennent ponctuer de grandes bouffées d'air ma vie de poète et me réconfortent dans les moments difficiles de l'écriture en solitaire.

V. et I.: Vous citez fréquemment les écrivains que vous lisez. L'énoncé de Gracq, "en lisant en écrivant ", s'applique à merveille à votre écriture. Peut-on voir cette pratique comme une forme de collaboration? Comment la percevez-vous?

D. D.: Comme une autre manière d'échapper à certains effets néfastes de la solitude. Écrire seule, bien sûr, mais toujours accompagnée par des voix amies, métamorphosées en quelque sorte par l'écriture, dont certains accents ne me sont pas étrangers. Des voix qui ont accepté de courir des risques en abordant, chacune à leur manière, cette réalité complexe qui est aussi la mienne, la nôtre. Je me sens souvent aux prises avec ce qu'Anne Hébert appelle d'“étranges forces obscures" qui paralysent à la fois ma vie et mon écriture, et me donnent l'impression de tourner à vide ou plus précisément de m'enliser dans une langue remplie de ténèbres, une langue de la blessure, d'où ne pourra jamais surgir aucune étincelle. À ces moments-là, j'ai besoin de chercher ailleurs, dans les livres des autres, par exemple, des bribes de sens, la piste inattendue ou le chaînon manquant. Juste un peu d'air pour ne pas suffoquer. Une seule petite phrase parfois suffit à produire un peu de lumière au milieu des ténèbres, là où je ne l'attendais pas. Une seule petite phrase qui me protège contre moi-même. Écrire seule, bien sûr, mais en continuant à lire, à me sentir liée au monde, à sa pensée et à son mouvement, quitte à être bousculée en cours de route. N'est-ce pas là, comme l'écrivait encore Anne Hébert, le grand pouvoir de la poésie: celui de rompre la solitude?

V. et I.: Vous êtes d'abord poète. Vous avez publié de nombreux recueils de poésie. Si tant est qu'on puisse répondre à une question comme cellelà, pourquoi la poésie? 
D. D.: Je n'aurais pas pu répondre à cette question au moment où j'écrivais mes premiers recueils, les écrivant sans être en quête d'une voix qui soit vraiment la mienne, m'insérant platement - par souci de protection sans doute - dans une langue préfabriquée, aux accents poétiques complètement anonymes. J'ai mis du temps, beaucoup de temps à laisser tomber les résistances de plus en plus nombreuses, de plus en plus farouches, qui me tenaient à distance de moi-même, qui m'étouffaient, lentement me tuaient. À cette époque, la lecture du roman de Jacques Ferron Les roses sauvages m'a bouleversée. Je me reconnaissais dans cette femme perdue, absente d'elle-même, que le parfum des roses finira par rendre folle, et qui mourra, suffoquée, après avoir battu sa petite fille. Ces "roses sauvages", dévastatrices, j'ai pris conscience que je les portais en moi. Pour survivre, il me fallait donc agir au plus vite, en extraire les racines, changer de vie et de ville, renouer avec cette part oubliée de moimême, renouer surtout avec la littérature vivante, dérangeante, de laquelle je m'étais tenue à distance trop longtemps. Cette littérature, qui se faisait surtout aux Herbes rouges et à La barre du jour, était écrite par des poètes modernes, engagés dans leur histoire, qui rêvaient de transformer le monde par le langage. Ces jeunes poètes, ces "têtes chercheuses", pour reprendre l'expression de Paul Chamberland, je les ai d'abord rencontrés dans les livres de Philippe Haeck, L'action restreinte. De la littérature et Naissances. De l'écriture québécoise, deux œuvres essentielles qui m'ont remise au monde et vers lesquelles j'étais allée parce que Philippe n'était pas tout à fait un étranger: nous avions étudié à l'université au même moment. Après, j'ai lu tout ce que ces poètes avaient produit, découvrant du même coup l'écriture bouleversante des femmes. Sans elles, je sais que je n'écrirais pas aujourd'hui. C'est accompagnée par ces voix - celles de Madeleine Gagnon, de Nicole Brossard, de Louky Bersianik, de France Théoret, de Louise Cotnoir, d'Hélène Cixous, entre autres - que je suis véritablement entrée en poésie et c'est avec leur audace que j'ai pu écrire La promeneuse et l'oiseau. Dans le Journal, qui suit le récit poétique, on retrouve cette citation de France Théoret: "J'écris parce que j'ai peur et j'ai peur parce que j'écris ", et cette autre, d'Hélène Cixous : "elle était le désir d'elle-même se cherchant dans le noir avec une telle nécessité; l'innocente urgence de femme. "Cette urgente nécessité d'écrire au plus près de soi, de son désir, de sa peur, de sa violence, même quand on se sent égarée, dispersée, diluée dans le noir, je l'ai rencontrée dans des livres de femmes, qui m'ont à la fois éblouie et secouée, et je crois que ce sont les effets de cette nécessité, choc et ravissement, qui un jour m'ont fait choisir - si on peut encore parler de choix - la poésie.

V. et I. : Vous avez publié et de la poésie en vers et de la poésie en prose. Ce sont là deux façons de voir le poétique...

D. D.: Il est vrai que je me suis beaucoup promenée en poésie, hésitant souvent longtemps, au moment où je commençais un nouveau recueil, 
entre le vers et la prose. Avec Le saut de l'ange, je suis même allée plus loin, rédigeant une première version du texte en prose, puis une deuxième, en vers, avant de revenir dans une dernière, celle qui a été publiée, du côté de la prose. Dans ce cas-là, le passage par le vers m'aura permis de lire le texte autrement, de le resserrer, de l'épurer de tout ce qui me semblait alors trop prosaïque. Pourtant je sais que cette attirance vers le narratif, avec ce qu'il entraîne, personnages, espaces, temps, événements, tous mes livres en portent la trace, et plusieurs critiques qui les ont commentés ont parlé de la récurrence de cette tentation dans mon travail. Déjà en 1985, j'avais été invitée par Gérald Gaudet à participer à un numéro de la revue Estuaire, qui avait pour thème "La séduction du romanesque". Je dois d'ailleurs dire que cette invitation ne m'avait ni heurtée ni surprise. Au contraire, j'ai pris un grand plaisir à écrire ce court essai, qui a pour titre "Poème de la durée/Texte", dans lequel j'ai essayé de comprendre d'où me venait cette attirance, en revenant en arrière, vers La promeneuse et l'oiseau, vers L'écran, et en faisant un détour par la peinture de Francine Simonin, par cette idée de la série que je retrouvais dans Films d'intérieurs. Les dessins et les tableaux de cette exposition présentée à la galerie Treize, cette année-là, m’apparaissaient comme une belle illustration de ce qu'on pourrait appeler la figuration narrative, c'est-à-dire un travail analogue à ce que je faisais en poésie; un travail en lutte contre cette sensation de fragmentation, de dispersion, tant de fois exprimée dans les livres de femmes. Citant dans mon texte cette phrase terrifiante de Roland Barthes, à propos de la poésie moderne: "La Nature y devient un discontinu d'objets solitaires et terribles, parce qu'ils n'ont que des liaisons virtuelles ", j'admettais du même coup mon effroi devant le pur poème, qui ne pourrait qu'accentuer cette impression de morcellement. Sans nier ni les ruptures, ni les déplacements, ni les retournements, je découvrais que j'étais à la recherche du fil de l'histoire, de ce fil que je ne pourrais retrouver que grâce à une voix - inscrite dans la mouvance d'une forme - qui, récitant, bouge, se déplace, fait des fouilles, en quête d'une vraie continuité, prenant cependant en compte les différents niveaux de réalité, les espaces fractionnés où des images se sont incrustées à notre insu. Cela dit, j'ai été de nouveau tentée par la poésie en vers dans Un livre de Kafka à la main, Mais la menace est une belle extravagance et Black Words, mais j'y suis chaque fois revenue avec ce désir, dont je parlais plus haut, de l'épuration, du dépouillement, après quelques livres à l'écriture plus débridée.

V. et I.: Tout récemment, après plus de vingt ans de publication, vous avez fait paraître votre premier récit. J'aurais envie de vous demander: d'où vous est v́enue cette nécessité de passer au récit?

D. D.: Sans doute m'est-elle venue de cette séduction que n'a jamais cessé d'exercer sur moi le romanesque et, d'une manière plus concrète, du plaisir que j'ai éprouvé à écrire des dramatiques, par exemple, ou 
même Cimetières : la rage muette, où quelque chose d'extérieur à la poésie a surgi, qui m'a envoûtée. Une réelle ouverture sur l'univers de la fiction, le beau mensonge de la fiction, et ce, malgré la part importante d'autobiographie qui s'y trouve. Or, après quelques tentatives insatisfaisantes, quelques mauvais débuts de roman où je ne suis pas arrivée à trouver la voix, celle qu'il m'aurait fallu pour me rendre jusqu'au bout, celle que j'aurais pu soutenir longtemps, sur presque deux cents pages, j'ai mis de côté mon désir. Jusqu'au jour où Jean Royer, alors directeur littéraire aux Éditions de l'Hexagone, m'a parlé de l'écriture d'un récit. Ce fut une sorte d'illumination. J'ai vite senti que le récit me donnerait la possibilité d'aller vers la fiction sans avoir à m'y installer de plain-pied dès le départ; que je pourrais plus facilement y trouver la voix, une voix de l'intime - familière malgré son étrangeté —, tout près de celle du poète, une voix qui ne m'offrirait pas autant de résistances que celle que j'avais cherché en vain à inventer hors de moi.

V. et I.: Mais votre récit garde tout de même des traces du poétique. Il s'agit bien d'un récit de poète...

D. D.: Oui, un récit de poète, dans lequel j'ai voulu poursuivre autrement, dans une autre forme, une exploration de sens... de langage, ce que j'avais déjà tenté de faire quand je me suis aventurée du côté du théâtre radiophonique. Dans "Ce que je cherche à faire", le texte que j'ai écrit pour De l'écriture du poème, un essai collectif dirigé par Jean Duval et publié par le groupe de création Estuaire, je citais cette phrase de $\mathrm{Na}$ thalie Sarraute, qui m'a accompagnée tout au long de l'écriture du récit: "Le lien indissoluble entre la réalité inconnue et la forme neuve qui la crée fait que toute exploration de cette réalité constitue une exploration de langage. " Dès le départ, contrairement à ce qui se passe en poésie, j'ai dû me méfier de ce qu'il y avait de trop superficiellement connu dans cette réalité de l'enfance, résister à l'attrait de l'anecdotique, me dépayser en quelque sorte, pour que l'exploration puisse avoir lieu et me permette d'entrer dans ce lieu trouble où paradoxalement deuil et Bonheur marchent côte à côte.

V. et I. : Que vos textes soient en vers ou en prose, on retrouve chez vous une constante: la quête de l'intime, qui a à voir chez vous avec l'autobiographie. Quel lien faites-vous entre l'intime et l'autobiographique?

D. D. : Je suis continûment aux prises, dans mon travail d'écrivaine, avec les liens étroits qui unissent au départ ces deux notions, souvent perçues comme interchangeables, presque synonymiques. Dans Vedute, le critique d'art René Payant, commentant deux expositions, Tridimension-Elles et Art et féminisme, aborde cette question avec beaucoup de justesse dans un chapitre qui a pour titre "L'intime comme lieu". "Si la biographie, la subjectivité, est partie de l'autobiographie et oriente sa cohérence comme récit, écrit-il, l'intime est vraiment dans l'écriture même qui construit le 
récit, là où le sujet s'échappe, déborde de lui-même. "Le mot est là : "écriture", celui auquel je m'accroche depuis toujours, qui me stimule et me permet d'espérer échapper, à chaque livre, à ce qu'on pourrait appeler l'intimisme, mot que je déteste peut-être autant que Barthes détestait le mot "familiarisme". Mon travail consiste à tenter cette expérience de l'échappement, du débordement de moi-même, à me délester de ce qui est trop proprement subjectif pour ne conserver, comme l'écrit encore Payant, "que les traces de l'affolement, du désir éveillé par l'intime à vouloir s'exprimer, s'imprimer dans des signifiants ". Avec l'espoir que ce qui s'imprime soit, chaque fois, une petite part de cette mystérieuse humanité que l'on porte, tous et toutes, en soi et qu'on ne peut abandonner à ellemême sans risque.

V. et I. : Dans Lettres à Cassandre, publié en collaboration avec AnneMarie Alonzo, vous vous posez la question du texte autobiographique, de sa pertinence. Se trouve affirmée là la nécessité d'une distanciation avec soi-même, même dans le texte autobiographique. Comment y tendezvous, dans votre écriture?

D. D.: Comme je l'ai déjà écrit ailleurs, y compris dans Lettres à Cassandre, j'ai longtemps eu des réticences à avouer l'importance de l'autobiographie dans mon travail. Avec le temps, j'ai compris que, comme la plupart des poètes, je poursuivais, de livre en livre, quelques obsessions qui ont partie liée avec ma vie - qui sont à l'origine même de mon désir d'écrire -, et que la vraie question à laquelle je devais répondre était celle-ci : comment faire surgir de ce qu'il y a de plus subjectif, de plus inavouable peut-être, une langue poétique, qui s'éloigne de l'usage courant, et qui en même temps rejoigne l'autre, les autres aux prises avec leur propre intimité fragile et l'opacité d'un monde dont l'air est souvent irrespirable? Ni mes grandeurs ni mes misères ne m'appartiennent en propre. Plusieurs écrivains, qui ont stimulé ma pensée et mon écriture, m'ont amenée à saisir que la vie intime, dégagée de ses caractéristiques trop personnelles, plutôt considérée comme un lieu secret, profondément humain, où de multiples mémoires s'affrontent et se confrontent, que cette' vie intime se retrouve en chacun et chacune de nous. C'est à cette humanité si souvent fragile, frileuse, inconséquente, qui est la nôtre, que je m'intéresse en écriture, à partir d'un lieu précis, le mien, celui qui m'est le plus facilement accessible. C'est là que se situent mon engagement et mon utopie.

V. et I. : En fait, on sent que toute votre pratique gravite autour de l'enfance et, au centre de l'enfance, de la mort de votre père, comme un trou qui aspirerait l'écriture...

D. D.: Oui, l'enfance resurgit toujours et partout parce que, comme je l'écrivais dans Leçons de Venise, je porte en moi une enfant inconsolable, confrontée très tôt aux multiples visages de la mort et néanmoins longtemps bercée, dorlotée, protégée sous un drap de Bonheur, tenue à dis- 
tance du deuil. Il y a, en effet, à l'origine, cette mort de mon père, mais je crois que ce sont les effets nocifs, dévastateurs à long terme, de cette mort sur ma mère, sur sa vie, et tout naturellement sur la mienne, qui m'ont ramenée vers cette mort, comme vers l'événement fondateur, originaire, à partir duquel tout s'est enclenché. Il me semble que ce ne soit pas tant l'absence du père, elle-même, qui ait enténébré à ce point ma pensée et mon émotion que la présence sans cesse inquiète d'une mère mélancolique, aux prises avec ce que Freud nommait le "regret de quelque chose de perdu ", un regret tel qu'il entraîne à la fois un sentiment de culpabilité et une sorte de désespoir sans fond. Dans ce contexte, aucun deuil n'est possible. Ni pour la mère, cela va de soi, ni pour la fille. Cette vérité évidente - et ses conséquences dans tous les replis d'une existence - , je ne l'ai découverte que lentement, souvent par bribes, en avançant à tâtons dans l'ombre, grâce à l'écriture poétique.

V. et I. : Cet événement est abordé dans La promeneuse et l'oiseau, dans le texte "La Blessure", publié dans Un livre de Kafka à la main, puis dans votre récit, Ce fauve, le Bonbeur... Mais dans votre récit, on sent que l'événement est moins douloureux, que le deuil est plus assumé... Est-ce le temps qui a passé? Ou le récit, qui permet de mettre les événements à distance? Comment voyez-vous cela?

D. D.: En effet, de texte en texte, la tonalité a changé, passant, de La promeneuse et l'oiseau à "La blessure ", du tragique au pathétique ou au lyrique, pour se présenter, dans Ce fauve, le Bonbeur - qui vient plus tard -, dépouillée de ce fatras, comme plus intimiste, plus près de cette tristesse que la petite fille ne pouvait pas nommer, mais qui l'habitait au quotidien. Dans sa critique du récit, Robert Chartrand écrivait : "Ni moyenne, ni exemplaire, c'est une enfance particulière, revisitée sur le ton de la confidence chuchotée. "Cela me paraît tout à fait juste. Or, pour que ce ton de la confidence soit possible, il a certes fallu que le temps passe; que, de livre en livre, l'écriture opère son lent travail de dévoilement, de "déchiffrement ", écrivait Marguerite Duras, permettant ainsi à l'archéologue de croire encore à l'utilité et à la nécessité de ses fouilles... en terrain miné. De temps en temps, une éclaircie, et la passion, et l'écriture se remettent en marche. Cependant, en ce qui me concerne, je ne crois pas que ce soit le récit, comme genre, qui ait permis cette mise à distance. Je dirais plutôt qu'en 1995 ou 1996 - plus de quinze ans après La promeneuse - les conditions avaient changé, étaient en quelque sorte favorables, que j'étais alors prête à m'aventurer de ce côté-là, à prendre ce risque. Certaines pages, entre autres, du Saut de l'ange et de Cimetières: la rage muette, certains textes de réflexion aussi, m'y avaient préparée. Je pense ici, en particulier, au texte de Cimetières, dans lequel la petite fille voit, par le trou de la serrure, sa mère pleurer; texte dont le ton annonce celui de Ce fauve, le Bonheur et d'où j'ai extrait cette phrase, "Nous le rejoindrons un jour", reprise dans le récit. Je pense également à 
la communication que j'avais lue au onzième colloque de l'Académie des lettres du Québec, qui avait pour thème "Les livres qui vous ont faits ", et à laquelle je suis revenue au moment d'écrire le chapitre "La grand-mère" C'est ma grand-mère maternelle - dont le discours janséniste niait sans cesse le corps que son souffle dans mon cou et sa voix réhabilitaient pourtant avec force - qui m'a servi de modèle.

V. et I. : Sans avoir signé de recueil d'essais - du moins pas encore vous avez pourtant écrit de nombreux textes de réflexion sur l'écriture. D'ailleurs, la réflexion est constamment présente dans votre poésie et dans votre récit. Comme chez beaucoup d'écrivains actuels, la réflexion chez vous semble faire partie de votre écriture.

D. D. : Elle m'est absolument essentielle. J'écris depuis toujours, avec cette impression, difficile à supporter et à accepter, d'être à la fois chargée et encerclée d'ombres, d'avancer dans le langage, en piétinant, en m'y égarant parfois. C'est pourquoi j'ai eu tant de fois recours, comme pour me ramener à l'ordre, à cette citation de l'écrivaine portugaise, Maria Gabriela Llansol: "Si l'on pousse assez loin dans le langage, on se trouve pris dans l'étreinte de la pensée." Or, pour arriver à "pousse[r] assez loin ", j'ai besoin de freiner l'avancée des émotions incontrôlées qui embrouillent tout, y compris le langage. Pour éviter la dilution, l'enlisement ou la fuite dans une poésie effrénée, pleine de lyrisme et d'inutiles épanchements, je dois me surveiller - "je me tiens à l'œil ", écrivait France Théoret. Je sais qu'on ne peut pas parler nä̈vement de l'intime, de tout ce qui sommeille ou s'agite au plus profond de soi, de l'inavouable, ni des liens obscurs, souvent tordus ou menaçants, qui existent entre cet intime et le monde, ni de lucidité, ni d'utopie. Avec le temps, j'arrive mal à concevoir une véritable démarche en écriture, ce corps à corps avec sa blessure autant qu'avec la langue, qui soit sans aucun lien avec la réflexion. La poésie, comme la vie, ne va pas de soi. Elle implique une pensée en mouvement, un travail... que les textes plus réflexifs, présentés à des rencontres d'écrivains ou à des colloques, viennent stimuler.

V. et I. : On vous a située dans le courant de la modernité, au Québec, tel qu'il s'est développé autour de La nouvelle barre du jour. Vous avez même fait partie du comité de rédaction de La nouvelle barre du jour. Quel bilan faites-vous aujourd'hui de cette expérience?

D. D.: Elle m'a tenue en éveil, poussée vers l'audace et le renouvellement, et souvent empêchée de céder à des impulsions trop lyriques. J'ai sans doute besoin d'être confrontée à des écritures différentes de la mienne, à des textes où le travail formel, avec sa rigueur et parfois même sa radicalité, occupe le premier plan, où le plaisir du jeu semble quelquefois l'emporter sur l'angoisse des obsessions. Ces écritures sont un contrepoids à mon penchant naturel et me ramènent tout droit à cette nécessité de l'exploration et du mouvement, du dépaysement. C'est à cause de cela 
que je m'intéresse, depuis quelques années, au travail de plusieurs écrivains - Michelle Grangaud, entre autres - qui publient chez P.O.L. Quant à La nouvelle barre du jour où j'ai travaillé pendant près de six ans, elle m'aura donné la chance de vivre cette modernité de l'intérieur de m'impliquer pour vrai dans cette aventure, de faire des choix; de vivre aussi cette euphorie des projets fous, démesurés, comme "Installations/Fictions " qui regroupait vingt-trois écrivains et écrivaines, et vingttrois artistes; de vivre tout cela, avec des écrivains, Normand de Bellefeuille et Marcel Labine, dont j'aimais les textes, la pensée, la passion et jusqu'à l'humour... un humour qui nous faisait du bien dans les moments difficiles - il y en a eu quelques-uns - où, épuisés par nos huit numéros par année, nous remettions tout en question, y compris la poésie, notre goût pour la poésie.

V. et I. : L'écriture de la modernité, au Québec, a été fortement marquée par la présence des femmes. Vous-même avez déjà affirmé, dans des réflexions sur l'écriture, votre recherche d'une subjectivité féminine, ce qu'on voit très bien quand on vous lit. Où avez-vous l'impression de vous situer dans ce courant?

D. D.: Je fais partie de cette génération d'écrivaines qui ont, comme le notaient Nicole Brossard et Lisette Girouard dans l'introduction de leur Anthologie de la poésie des femmes au Québec, "une mémoire d'avant le féminisme [et qui] trouvent en ce dernier un écho à leur expérience ". Et, en ce sens, je me sens concernée par le texte que vous avez fait paraitre dans la revue Trois, "Là d'où je viens: notes sur l'écriture et le féminisme", parce que je viens d'un lieu qui ressemble étrangement à celui que vous évoquez. Ce Québec d'avant la Révolution tranquille, cet œil menaçant de Dieu, cette "noirceur pesante" de l'enfance, cette négation du corps autant que de la voix, cet effrayant silence des femmes, et leurs désirs enfouis, et leurs peurs, et leur effacement, toute cette mémoire, je la porte au fond de moi. Sans la prise de conscience des femmes qui m'ont précédée et accompagnée, je crois que je n'aurais jamais eu accès à cette mémoire. $\mathrm{Ni}$, au bout du compte, à ma pensée, à mon imaginaire, à ma voix. Aujourd'hui je sais que j'écris avec cette conscience heureuse mais toujours vulnérable, inquiète, d'être une femme qui écrit, une femme parmi d'autres et cependant unique, avec sa fougue, sa passion et sa langue qui n'a par moments, il faut bien le dire, rien de maternel.

V. et I. : En vous lisant, on voit que ce questionnement passe souvent par la relation à la mère...

D. D.: En 1979, dès ma première lecture de Et l'une ne bouge pas sans l'autre de Luce Irigaray, j'ai été remuée. Chaque mot a suscité en moi un vif écho, à cause de cette relation très particulière que j'ai vécue avec ma mère, une mère plus que protectrice, qui croyait en toute bonne foi tenir entre ses mains ma vie et mon salut, pendant toutes ces années qui ont 
suivi la mort de mon père. En une quinzaine de petites pages, d'une poésie troublante, l'écrivaine présente cette relation mère-fille comme naturellement étouffante, mortifère même, ne pouvant d'aucune façon mener à une vraie rencontre de deux vivantes, liées par une affection réciproque mais libres, détachées l'une de l'autre. Du moins, tant que les mères ne changeraient pas. Dès cette première lecture, j'ai su que je ne pourrais pas y échapper: j'écrirais à partir de là, de ce nœud, puisqu'il y allait de ma vie. Écrire pour vivre mieux, vivre vraiment, réconciliée un jour avec moi-même et avec ma mère; écrire aussi en pensant à elle, à sa solitude, en cherchant à mettre des mots sur ses silences.

V. et I.: Votre récit fait un lien très intéressant entre mère, deuil et Bonheur... En fait, vous revendiquez le droit à la souffrance, qui est aussi le droit à la vie...

D. D.: Ce retour sur mon enfance m'aura ouvert les yeux. On ne peut nier ce droit à la souffrance ni ce droit à la vie sans que fatalement, par la suite, se répercutent, dans chacun de nos gestes et chacune de nos émotions, les effets néfastes de cette dépossession. Et ce mot "dépossession" me ramène à ce qui a été mon livre de voûte, Le torrent. Je suis toujours fascinée par cette façon qu'ont nos premières intuitions de ne pas se perdre, de resurgir à l'improviste, plusieurs années plus tard, sous forme d'évidences: par une volonté antérieure à la mienne, j'ai été une enfant dépossédée du monde. Or, cette affirmation, j'ai mis beaucoup de temps à la faire mienne, à la reformuler au féminin, malgré mes multiples relectures du Torrent. Sans doute faut-il beaucoup de désir et de patience pour arriver à entendre ce qui a été si longtemps refoulé et ce qui, aujourd'hui encore, ne sort de l'ombre que par bribes furtives. Mais cette passion pour la lumière me vient de là : c'est parce que je l'avais entrevue dans les premières œuvres d'Anne Hébert que j'ai éprouvé le désir de partir à sa recherche, malgré les exigences insatiables du Bonheur, dont les effets continuaient de me hanter et qui ont souvent freiné ma ferveur.

V. et I. : Deux ans après Ce fauve, le Bonheur, vous êtes revenue à la poésie, l'automne dernier, avec Tombeau de Lou. Comment ce retour s'est-il fait?

D. D.: Après le récit, ce retour à la poésie, je le souhaitais, le prévoyais même, mais... autrement que sous la forme d'un tombeau. Les photographies, qui accompagnent le texte, Alain Laframboise me les avait offertes - format réduit - le 3 avril 1997, jour de son vernissage à la Galerie Graff. Un an plus tard, je recevais une bourse du Conseil des arts et des lettres pour un projet d'écriture poétique, qui les impliquait et dans lequel je mettais l'accent sur leur étrange pouvoir de séduction. Ces "visions domestiques " m'avaient placée, avant même que les mots ne viennent, face à certaines émotions, que je n'avais encore jamais abordées d'une manière explicite dans mon travail. Pendant cette année de réflexion, mes 
obsessions d'écrivaine - enfance, arrachement, cri, mort, Bonheur à tout prix - s'étaient subtilement avancées vers moi, marquées par une sensualité particulière, jusque-là interdite. J'avais la conviction que quelque chose d'inédit était en train de se passer. Or, en décembre 1998, une mort tout à fait inattendue a bouleversé ma vie. L'amie de toujours, celle qui avait servi de modèle à l'Anouk de La promeneuse, à la Lou de "La blessure " et de Cefauve, le Bonbeur, a été emportée par un cancer des os, en deux mois et demi. Cette mort était impensable, inadmissible, car cette amie était ma sœur choisie. Présente partout, jusque dans mon écriture, comme la figure amie faisant contrepoids à celle de la mère. Nous nous étions connues à l'âge de cinq ans, l'année de la mort de mon père, et nous avions pris l'habitude de nous répéter que nous allions vieillir ensemble, nous accompagner, l'une et l'autre, dans la vieillesse. Je sais depuis longtemps que l'éternité n'existe pas, mais nous en avions inventé une, bien terrestre, qui nous liait l'une à l'autre. Bizarrement, après cet événement, les photographies d'Alain Laframboise me sont apparues comme prémonitoires, chargées de mélancolie, dans la mesure, entre autres, où s'y côtoient enfance et mort : petits cercueils et tricycle jaune, poupées jumelles aux chapeaux rouge et blanc, stèles et croix, etc.

V. et I. : Quels sont vos projets? Reviendrez-vous au récit éventuellement?

D. D. : Peut-être un jour. Avant j'aurais plutôt envie de reprendre la narratrice de Ce fauve, le Bonbeur - là où elle s'est arrêtée, en 1960 -, d'en faire un personnage de roman, et ce, afin d'arriver à m'en distancier davantage. Mais, pour le moment, je travaille à un autre texte poétique, fortement marqué par mon incursion dans la prose bien qu'il soit écrit en vers. Dans ce long poème, une femme adresse à sa mère, vieillissante et souffrante, une lettre où les préoccupations présentes, la réalité immédiate, le voyage et l'éloignement, les arbres, la maladie, la mort, l'écriture, l'emportent sur le passé évoqué à quelques reprises. À l'origine de ce poème, il y a la maladie de ma mère, son départ pour l'hôpital et finalement son installation dans une résidence pour personnes âgées. Il y a surtout la mort la frôlant de plus en plus souvent, la mort quasi quotidienne et chaque fois réversible. D'où ce titre Pendant la mort. C'est-à-dire pendant que la mort s'éprouve, se pose dans chaque parcelle du corps de la mère et, par voie de conséquence, de la fille, c'est-à-dire avant la vraie fin, la fin définitive. Qu'advient-il d'elles pendant cette interminable mort? Je me suis intéressée ici au présent de cette mère et de cette fille, toutes deux vieillissantes et conscientes de leur vieillissement, toutes deux porteuses d'une mémoire inoubliable - entachée par la mélancolie contagieuse de la mère - , mais toutes deux encore emportées par le mouvement irrésistible de la vie. Et néanmoins différentes, éloignées l'une de l'autre par leurs croyances, leurs valeurs, leur culture, leurs rêves, leur regard sur le monde, leur langage, le temps, etc. Quelques pages - d'un premier état de ce texte - ont été publiées dans le numéro 79 de la 
revue Mobius, préparé par Lysanne Langevin, sous le titre de "Tu vieillis de plus en plus vite, et je te suis de près ". Le relisant au moment de la publication, j'ai pris conscience de son originalité - si je le compare à l'ensemble de mon travail - et j'ai éprouvé le besoin de le mener plus loin, de m'en servir comme point de départ d'une nouvelle approche de cette relation mère-fille, qui s'écrirait cette fois au présent.

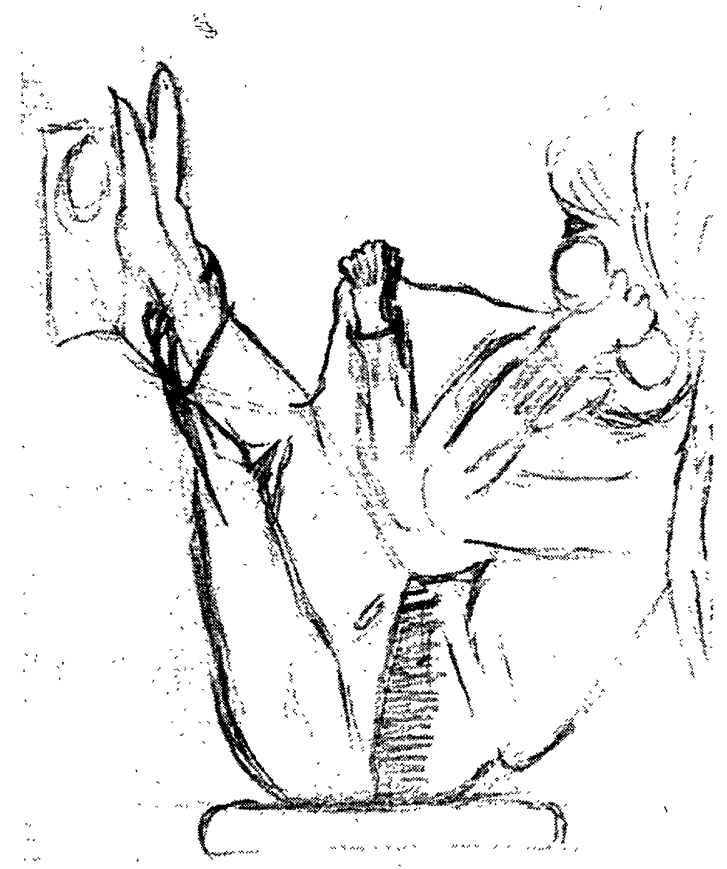

Portrait de l'auteure

par sa mère Simonne Brousseau Desautels, circa 1959 